\title{
Analysis of an Adaptive Switching Point for LTE TDD by Dynamic System-Level Simulations
}

\author{
Christian Backfrieder and Gerald Ostermayer
}

\begin{abstract}
This paper introduces an LTE system level simulator and presents investigations of scenarios with differently balanced traffic emergence between uplink and downlink as well as inhomogeneous distribution of link direction asymmetry. In the TDD mode of LTE, duplexing is implemented via sharing the time domain between uplink and downlink users. This is done by different switching configurations, which offer varying capacity distributions between downlink and uplink, from a downlinkheavy configuration with 90 percent of the capacity in downlink direction to uplink heavy configurations with 60 percent uplink. We utilize the available capacity perfectly by adjusting the link configuration appropriately to traffic emergence. Performance gains when selecting a configuration appropriate to the arising traffic compared to usage of a static configuration with equally distributed capacity are investigated. In case of different configurations, additional interference can occur in neighbored cells due to distinct communication direction at the same time. We clarify the differences between these scenarios and quantify the gains and losses when the switching point between uplink and downlink is variable.
\end{abstract}

Keywords-mobile network simulation, system level, long term evolution, time division duplex, variable switching point

\section{Introduction And PRoblem Description}

$\mathbf{R}$ ADIO resource management is a very important part in mobile cellular networks when looking at the overall performance of the system. This work focuses on the Time Division Duplex (TDD) mode of 3GPP Long Term Evolution (LTE), where the same frequency band is used for Uplink (UL) and Downlink (DL) transmission. This way, the reserved frequency band can be used more efficiently, because no bandwidth is wasted if traffic is balanced unequal between uplink and downlink. Figure 1 shows an example for bandwidth usage with asymmetric arrangement between uplink and downlink users. The upper part shows a fixed switching point, i.e. the same amount of time resources are available for both downlink and uplink traffic. In the lower part, the advantage of TDD is utilized, where the available bandwidth amount is spread unequal between the two link directions. In LTE, cells do not switch transmission direction arbitrarily on demand, but use predefined frame configurations which specify at which point in time downlink or uplink users are to be scheduled. Due to current applications for mobile networks such as consumption of audio and video streaming from sharing platforms, web

C. Backfrieder is with the Research Group Networks and Mobility, Department of Mobile Computing at the University of Applied Sciences Upper Austria, Campus Hagenberg, Softwarepark 11, 4232 Hagenberg, Austria (e-mail: christian.backfrieder@fh-hagenberg.at).

G. Ostermayer is with the Department of Mobile Computing at the University of Applied Sciences Upper Austria, School of Informatics, Communications and Media, Softwarepark 11, 4232 Hagenberg, Austria (e-mail: gerald.ostermayer@fh-hagenberg.at).

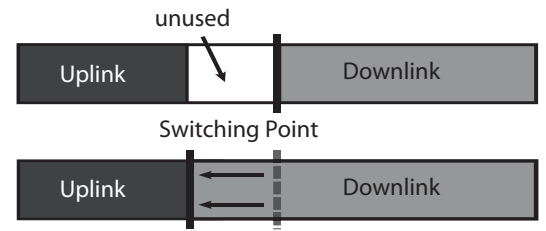

Fig. 1. Adaptive TDD switching point

browsing including data up- and download or video calls, traffic may be distributed unequally between UL and DL. Since the traffic emergence can also vary spatially from Base Station (BS) to BS, it is suggestive that different cells use different link configurations to react to this inhomogeneity. Preferably, these configurations match the User Equipment (UE)'s demands as good as possible and provide appropriate resources depending on the current traffic situation.

This paper introduces a simulation tool which provides the flexibility to simulate with differently defined link configurations for each BS. The produced simulation results include comparisons between variable duplex configurations with diverging traffic emergence and show that adjustment of the available capacity is beneficial for both operators and users.

The rest of this paper is structured as follows. The next chapter gives an overview of related work. The basic parts of the simulation environment on system level are explained in chapter III. Moreover, the particular influence factors on the results are elucidated. In the fourth chapter an introduction of LTE TDD mode is given, as well as resource scheduling and handling of the users needs. Chapter V explains the particulars of the used system model. The sixth chapter finally points out the simulation results, how different parameters of UL and DL configurations impact the capacity and other performance indicators for operators, followed by a conclusion.

\section{RELATED WORK}

Numerous projects and publications related to LTE and LTE Advanced (LTE-A), simulation and performance evaluations exist in literature. This section shortly describes some relevant approaches. Extensive simulators with many configuration possibilities and applications are presented in [1] and [2]. However, the capabilities for configuration of the duplexing mode to TDD and also dynamic selection for an appropriate configuration are limited. Zheng et. al. present Open-LTE, which is a simulator focused on performance measurements for video streaming [3]. A vital issue also for this simulation, but out of scope of the topic for this paper is synchronization of the network [4]. Furthermore, investigations on capacity distribution between UL and DL were done by Wang et. al. [5]. 
The higher demand for DL capacity was solved through using dedicated DL cells, while UL traffic was served by microcells with lower throughput

Our simulation framework has a strong focus on investigations of appropriate selection of a link duplex configuration in LTE TDD and quantifies throughput comparisons with or without capacity adjustments shifted between UL and DL.

\section{Simulation EnVironment}

When simulating whole mobile networks, the simulation is often split into several parts due to performance reasons, consisting of link-level and system-level simulations [6]. The link level simulations consider influences at single transmission channels and produce representative mapping curves that are used in system level for modeling this channel. To analyze the performance of whole networks, meaning throughput over time, coverage, cell planning or interference, all these influences have to be modeled as a system level [7]. This simulator was specially developed for LTE networks, but the basic part is designed very generic in order to allow usage for other types of cellular networks as well. The framework includes modeling of user movement, pathloss calculation, impacts of slow and fast fading, handovers, configurable cell layouts and graphical output and can be categorized in several parts.

Modeling of channel effects strongly influences results and is therefore essential in order to achieve results closely related to the real world. The following models are used in our simulator.

\section{A. Geometric Pathloss}

Within the scope of this paper, we implemented two models, applicable for rural and urban scenarios in [8] and [9] respectively, since loss behavior diverges significantly depending on the environment.

\section{B. Slow Fading}

In order to achieve local correlation of slow fading values, we use a two-dimensional pregenerated slow fading matrix, which is created according to [10]. The size of this map must be at least as big as the simulation area extended by two times the correlation distance. For each base station, the slow fading map is shifted by the correlation distance to achieve maps which are uncorrelated among each other while still using the same map for all independent connections.

\section{Fast Fading}

As done for slow fading, we also use a pre-generated one dimensional Rayleigh faded array. For modeling different speeds, the access index is scaled accordingly. In order to achieve apparently independent fast fading behavior, the index for each connection is initially diced randomly.

\begin{tabular}{c|rlllllllllllll} 
UL- $D$ L & UL-DL switch & \multicolumn{10}{c}{ Subframe Number } \\
Configuration & periodicity & $\mathbf{0}$ & $\mathbf{1}$ & $\mathbf{2}$ & $\mathbf{3}$ & $\mathbf{4}$ & $\mathbf{5}$ & $\mathbf{6}$ & $\mathbf{7}$ & $\mathbf{8}$ & $\mathbf{9}$ \\
\hline 1 & $5 \mathrm{~ms}$ & D & S & U & U & U & D & S & U & U & U \\
\hline 2 & $5 \mathrm{~ms}$ & D & S & U & U & D & D & S & U & U & D \\
\hline 3 & $5 \mathrm{~ms}$ & D & S & U & D & D & D & S & U & D & D \\
\hline 4 & $10 \mathrm{~ms}$ & D & S & U & U & U & D & D & D & D & D \\
\hline 5 & $10 \mathrm{~ms}$ & D & S & U & U & D & D & D & D & D & D \\
\hline 6 & $10 \mathrm{~ms}$ & D & S & U & D & D & D & D & D & D & D \\
\hline 7 & $5 \mathrm{~ms}$ & D & S & U & U & U & D & S & U & U & D
\end{tabular}

Fig. 2. Link Duplex Configurations in LTE TDD

\section{LTE Duplexing AND RESOURCE ASSIGNMENT}

Basically, it is obvious that traffic in a mobile network takes place as streams to and from the terminals in downand uplink direction respectively. Certainly, these streams must be separated from each other. The LTE standard does not restrict this method to only one possibility, but leaves it open for operators and hardware manufacturers how to implement duplexing [11]. One of the goals for this spectrum flexibility is to allow the deployment to fulfill different requirements for the network. This includes the duplex modes, as well as different arrangements and sizes of the available spectrum.

\section{A. LTE Time Division Duplex}

The nonpaired frequency band is shared in time between uplink and downlink, as well as between users. One frame consists of 10 subframes, which can be reserved for upor downlink. In case of asymmetric traffic emergence, a key advantage of LTE TDD is the possibility to select an appropriate link duplex configuration out of a predefined set, as shown in Figure 2. The duplex switching point is simply changed, and capacity is moved from downlink (D) to uplink (U) or reverse as desired. S marks a spare interval used as guard period for switching procedure.

\section{B. Data Scheduling and Algorithms}

The assignment of available resources is crucial in LTE networks. The scheduler gets the quality information of the users or measures them itself and allocates channels in time and frequency in a way to make the highest possible use out of the highly frequency selective channel. Our simulator supports the following schedulers.

1) Round Robin: This well-known algorithm is the simplest and least intelligent scheduling implementation. It assigns the same amount of resources (the same amount of time) to each communication link distributed equally. However, it does not take the current channel conditions into account, therefore the service quality can't be guaranteed and the overall performance of the system is decreased, compared to a Best-CQI or Max C/I scheduler [12, p.112].

2) Maximum Throughput: The goal is to maximize cell throughput, by ignoring fairness completely and assigning the whole bandwidth to the user(s) with the highest Channel Quality Indicator (CQI) value. Obviously, the maximum throughput (or also called Max C/I) scheduler might potentially not schedule users with constantly bad conditions at all, which causes starvation of cell edge users. 
3) Proportional Fair: While the round-robin scheduler does not take the current channel conditions into consideration, and maximum throughput causes starvation of cell edge users, a practical scheduler should operate somewhere between among these, as does the proportional fair scheduler. It favors users with good channel conditions, but also ensures that every user gets assigned at least a minimum amount of the available resources. This is implemented by assigning resources proportional to the reported CQI values. At each time instant, the requests are weighted with the reported quality index and resources are assigned according to equation (1):

$$
\text { channels }_{x}=\left\lfloor\frac{C Q I_{x}}{\sum_{n=0}^{N} C Q I_{n}}\right\rfloor \cdot \text { num }_{\text {channels }}
$$

The number of channels per user is channel $s_{x}, x$ is the user index, $N$ the number of users in the current cell and num $_{\text {channels }}$ the maximum channel amount in the current cell. By using the floor-function, it can occur that some channels are left unassigned after the operation from equation (1). These are assigned to users in order of scheduling requests equally distributed.

4) Best-CQI: The principle of this scheduler is to assign the available subcarriers equally distributed, but in contrast to the round robin scheduler it takes the instantaneous channel conditions of the users into account. Each channel is assigned preferably to UEs which report this channel as one with good conditions. Compared to round robin, higher throughputs can be reached while also being fair in terms of assigned transmission time.

\section{System Model Insights}

\section{A. Traffic Generation}

For reasons of simplicity, our simulator models endless and continuous availability of data for all users, both for UL and DL connections in order to keep system load constant. This service is comparable to up- or download of very large files. The overall number of users in the simulation is also stable, considering that each user tries to transmit data of infinite length. However, the distribution of UEs between UL and DL is variable.

\section{B. Channel-Dependent Scheduling for UL and DL}

The scheduling interface expects a CQI description for each user to be passed, independent of proximity to reality or how it was created. Limitations for CQI feedback are different in uplink and downlink. Besides that, channels can be assigned arbitrarily in DL, while only contiguous blocks can be assigned in UL direction. For simplification reasons, the same schedulers are used for UL and DL. Obviously, this does not imply the contiguousness-constraint for uplink channel assignment. In order to satisfy this requirement, the originally performed channel assignment from the selected scheduler is reordered in such a way that the best possible match of old and reordered assignment appears. In other words, the number of differences between the old and new assignment vector is minimized.

\section{Signal to Interference and Noise Ratio (SINR) Calculation}

The overall interference which impacts the SINR is comprised of multiple different sources. Intra-cell interference is omitted completely through usage of orthogonal transmission schemes (Single Carrier Frequency Division Multiple Access (SC-FDMA) and Orthogonal Frequency Division Multiplex (OFDM) respectively) for both uplink and downlink direction. Intra-cell interference between up- and downlink is obviously not possible in TDD mode. Hence, interference can only stem from other base stations or UEs in the surrounding area. A precondition for received interference is naturally that both transmissions must occur at the very time using the same time slot. Furthermore, it must be mentioned here that synchronicity in time domain of the whole network is assumed. This leads to Inter-Cell Interference (ICI) emergence in one timeslot at a maximum, while in a non-synchronized network, interference spans over two timeslots in the majority of cases.

In LTE TDD, multiple circumstances can implicate disadvantageous interference conditions, as Figure 3 shows. For the first two situations, it makes no difference if neighbored BSs use distinct link duplex configurations, interference may occur in either case. The BS and UE references in brackets refer to exemplary situations in Figure 3(b).

- BS-MS interference: UEs in DL mode $\left(\mathrm{UE}_{D}\right)$, which receive also signals from neighbored $\mathrm{BS}\left(\mathrm{BS}_{1}\right)$ transmitting to their assigned UEs $\left(\mathrm{UE}_{A}\right)$ also in DL mode

- MS-BS interference: BSs $\left(\mathrm{BS}_{2}\right)$, which receive not only the valuable signal from the UE in UL mode $\left(\mathrm{UE}_{E}\right)$, but also from transmitting $\mathrm{UEs}\left(\mathrm{UE}_{B}\right)$ from neighbor BSs

In case of selection of different link duplex configurations, also the following new situations may arise.

- BS-BS interference: DL connections $\left(\mathrm{BS}_{1} \rightarrow \mathrm{UE}_{A}\right)$ interfere with connections in UL direction $\left(\mathrm{UE}_{E} \rightarrow \mathrm{BS}_{2}\right)$, if $\mathrm{BS}_{1}$ uses the very same time slot for transmission when $\mathrm{BS}_{2}$ is receiving data.

- MS-MS interference: UL connections $\left(\mathrm{UE}_{E} \rightarrow \mathrm{BS}_{1}\right)$ interfere with connections in DL direction $\left(\mathrm{BS}_{3} \rightarrow \mathrm{UE}_{D}\right)$, also if both connections take place at the same time and frequency. Both MS may be located very close to each other, which is a fact that worsens mutual influence of both connections.

\section{Selection of Transmission Modulation and Coding Scheme (MCS)}

When scheduling of users is finished, the transmitted amount of data is determined. Therefore, a final MCS value must be selected by the scheduler which is then used for the transmission. This presupposes selection of a CQI value, which is then mapped to the MCS. In downlink direction, calculation of the transmission CQI is performed as described in Figure 4. The necessary mapping curves which abstract the link level are generated following [13] and [14]. This CQI value is provided for the scheduler in order to allow intelligent, channel dependent scheduling, taking the conditions of the respective UE into account. However, the scheduler needs not to stick to the given channel information - this is just a notification of the UE. 


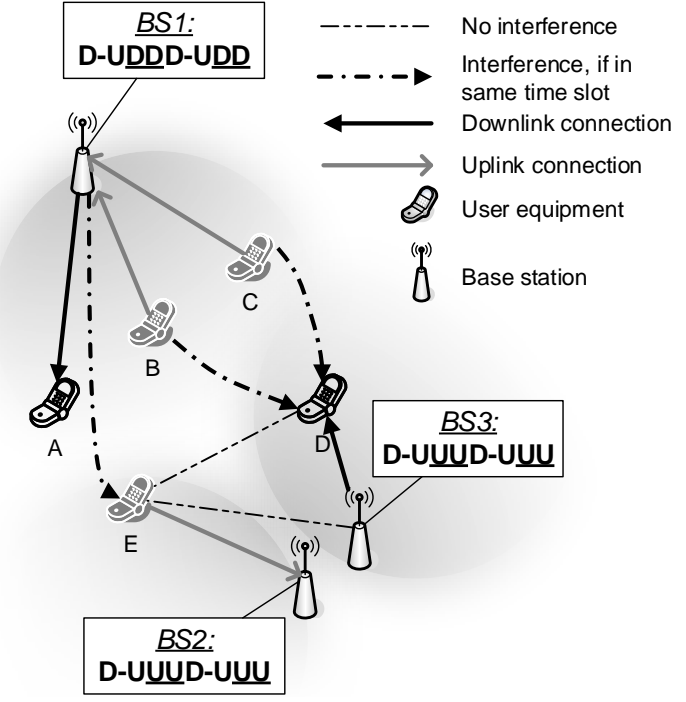

Fig. 3. Interference energence with varying TDD frame configuration

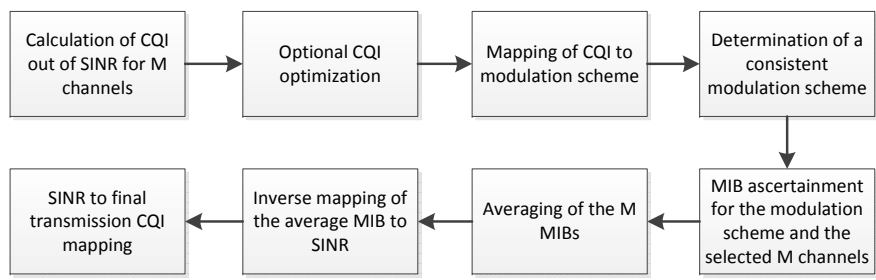

Fig. 4. Determination of final transmission CQI

Due to the divergence between the reported channels with the corresponding CQI value and the actually assigned channels, no current quality information is available for channels which were not reported. Hence, it is not suggestive to use the CQI value from the latest report, because this is likely to be higher than the quality for the actual channel assignment. Selection of too high CQI and MCS values derived therefrom leads to a high Block Error Rate (BLER) and no ideal bandwidth utilization. Therefore, the wideband CQI is consulted as a transmission CQI. In order to smooth away the averaging error, this CQI is optimized by considering transmission from the near past (section V-F).

The averaging for calculation of the wideband CQI transmitted by the UE that is used for transmission is done by the arithmetic mean, which is the best approach with the least difference to the optimal CQI, proved by simulations.

\section{E. SINR to CQI Mapping}

The mapping from SINR to CQI was simulated for a target BLER of the first transmission and one Physical Resource Block Pair (PRBP) of 10\%, which is an acceptable BLER target value for LTE networks $[15$, p. 2]. The mapping is dependent from the used link level, which is the reason why such a simulation is necessary and specific to the applied link level abstraction. This simulation was done by assuming a CQI value, mapping it to a modulation scheme (one of Quadrature Phase-Shift Keying (QPSK), Quadrature Amplitude Modulation with 16 symbols (16QAM) or Quadrature

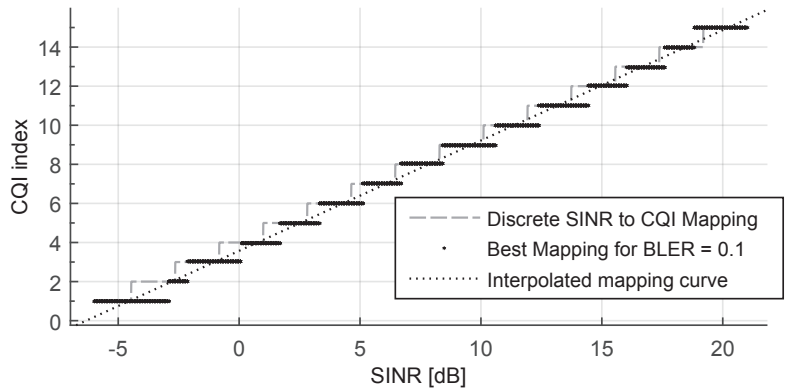

Fig. 5. Simulated SINR to CQI Mapping

TABLE I

CQI OPTIMIZATION SCHEME

\begin{tabular}{|cc|cc|}
\hline BLER (\%) & CQI Corr. & BLER & CQI Corr. \\
\hline$<5$ & +2 & {$[12$ 20[ } & -1 \\
{$\left[\begin{array}{c}5 \\
7\end{array}[\right.$} & +1 & {$[2035[$} & -2 \\
{$[712[$} & 0 & $>35$ & -4 \\
\hline
\end{tabular}

Amplitude Modulation with 64 symbols (64QAM)) and a code rate according to [16, p. 65] from $3^{\text {rd }}$ Generation Partnership Project (3GPP). Out of that, an MCS index is determined and therewith a Mutual Information (MI). In turn, a Mutual Information per Bit (MIB) is calculated by using this MI, which further acts as input to determine the BLER. The results are sets of BLER values for each given SINR, and such a set for each CQI value. For each CQI, the resulting BLER out of the set which is closest to $10 \%$ is consulted for the mapping curve. Figure 5 illustrates the simulation results, with the resulting BLER values that are closest to $10 \%$ depicted as solid black curve. For the final mapping function (dotdashed black curve), the single simulated points are interpolated linearly using a one-dimensional polynomial fit function. The dashed gray curve finally shows the used mapping from SINR to CQI for the used link level.

\section{F. CQI Optimization}

The scheduler needs not to stick to the CQI information from the UE, but can decide itself whether or not to correct or adapt these values. Due to the fact that not each UE can be allocated to use its preferred channels, natural deviations in preferred and real channels occur. Therefore, an optimization has been implemented in this simulator, which looks back a certain amount of time and adapts the reported CQI according to this information. The time was set to 40 transmissions in the past, which turned out to be a good balance between shortterm variations and a too high averaging error. The history data contains information about the success of the last 40 transmissions and implies a short-term BLER which is used to correct the transmission CQI value according to Table I.

The achieved gains are not only confined to the throughput, but also the average BLER (Figure 6). The throughput values are close to each other at an average of $5.88 \%$ (Figure 6(a)). The best gains for this simulation which included 17.86 users per cell at an average, were achieved for Best-CQI scheduler in both cell throughput and BLER. 


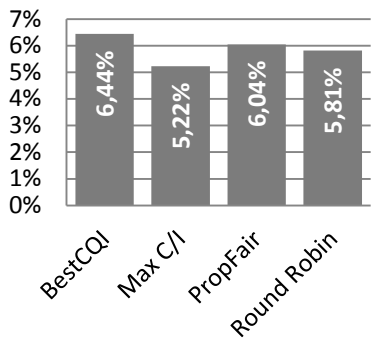

(a) Throughput Gain (percentage)

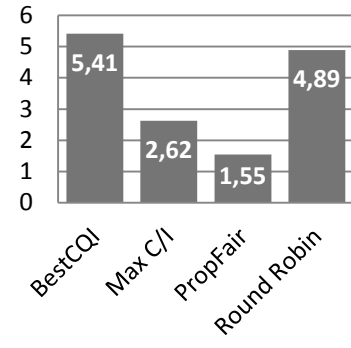

(b) BLER Gain $\frac{B L E R_{\text {old }}}{B L E R_{n e w}}$
Fig. 6. Bias of Throughput and BLER due to Optimization

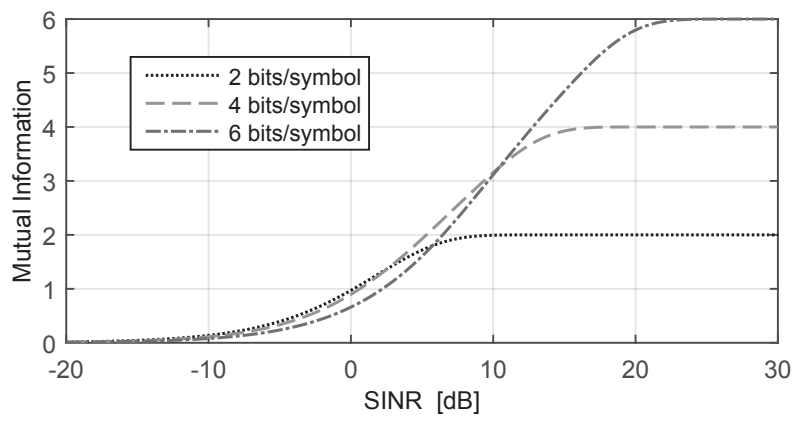

Fig. 7. SINR to MI Mapping for QPSK, 16QAM and 64QAM

\section{G. Mapping from CQI to Modulation Scheme and Code Rate}

According to Table 7.2.3-1 from [16, p. 65], the determined CQI value is turned into a modulation scheme (one of QPSK, 16QAM or 64QAM which can transmit 2, 4 or 6 bits per symbol respectively) and a code rate. The modulation scheme is needed for determining the MI, since the number of simultaneously transmitted bits influences it.

\section{H. Mapping from SINR to MI}

The MI for a transmission is determined by the mapping curves in Figure 7, depending on the SINR at the receiver. To calculate the MIB, this value simply must be divided by the number of bits that are transmitted by this symbol.

\section{Determination of Transmitted Bits}

On the basis of the transmission MCS and the number of Physical Resource Block (PRB)s per Codeword (CW), an exact number of bits transmitted in this $\mathrm{CW}$ can be determined by using mapping Tables from [16, p. 34-40]. A codeword, then, is a PRB with error protection. For each MCS, a mapping curve is provided with the appropriate modulation scheme applied. The number of transmitted bits rises with increasing CW size and MCS.

\section{J. Mapping of MIB to BLER}

A very important mapping is that from the determined MI and MIB calculated therefrom to a BLER. For the purpose of detecting the success of a whole transport block, a random value is diced for each PRB of the CW. The BLER mapping function returns the same amount of values, representing the error probability for the respective block. For a successful transmission, each of these diced values must be greater than

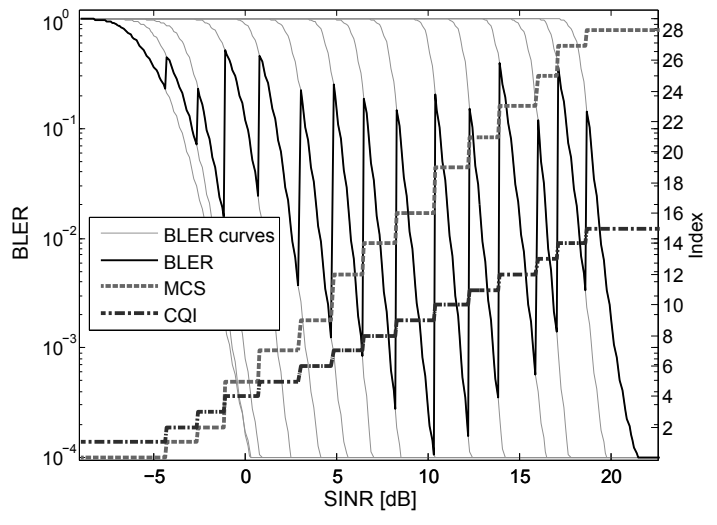

Fig. 8. Relation between SINR, MCS, CQI and BLER TABLE II

SIMULATION PARAMETERS

\begin{tabular}{|c|c|}
\hline Scheduler & Best CQI \\
Users in System & 1100 (fixed) \\
Number of Cells & 55 \\
Area of Investigation & $2200 \times 2200 \mathrm{~m}$ \\
Environment / Pathloss Model & Manhattan / Berg's Model \\
Sigma Slow Fading (SF) & $4 \mathrm{~dB}$ \\
Correlation Distance SF & $10 \mathrm{~m}$ \\
Fast Fading Resolution & $0.83 \mathrm{~mm}$ \\
Handover Margin & $4 \mathrm{~dB}$ \\
Maximal DL / UL Power & $46 \mathrm{dBm} / 23 \mathrm{dBm}$ \\
$P_{0}$ Correction for Uplink TPC & $-48 \mathrm{dBm}$ \\
$\alpha$ Correction for Uplink TPC & 0.5 \\
Antenna Gain & $16 \mathrm{~dB}$ \\
Antenna Tilt & $5 \mathrm{degrees}$ \\
Antenna Type & Omnidirectional \\
Bandwidth per Cell & MHz (50 channels) \\
M for CQI Reporting & 6 \\
CQI Optimization & on \\
&
\end{tabular}

the BLER probability. If so, the whole block is treated as successful. If not, the block fails and the next transmission of the Asynchronous Repeat Request (ARQ) mechanism is initiated. The ARQ allows combination of all transmissions, which results in a better BLER for the same MIB, i.e. a leftshift of the mapping curve.

\section{K. Relation between SINR and BLER}

When using the simulated mapping curve from SINR to CQI from section V-E, a BLER can be determined as described in section V-J. Figure 8 depicts the relation between CQI, MCS and BLER respectively. The light gray curves show the BLER for each CQI value. The thick, gray MCS curve shows the mapped MCS at a specific SINR. Resulting therefrom, a block error probability can be determined. The jumps of the curve occur due to the switch to the next higher MCS.

\section{AnAlysis and Simulation Results}

This section describes the simulation setup and results concerning TDD mode of LTE, which were achieved by using our system level simulator. In order to allow representative comparison, all simulations described in this section were done using the same basic parameter set (system users, environment, Transmit Power Control (TPC), antennas, scheduling algorithm) as specified in Table II. 


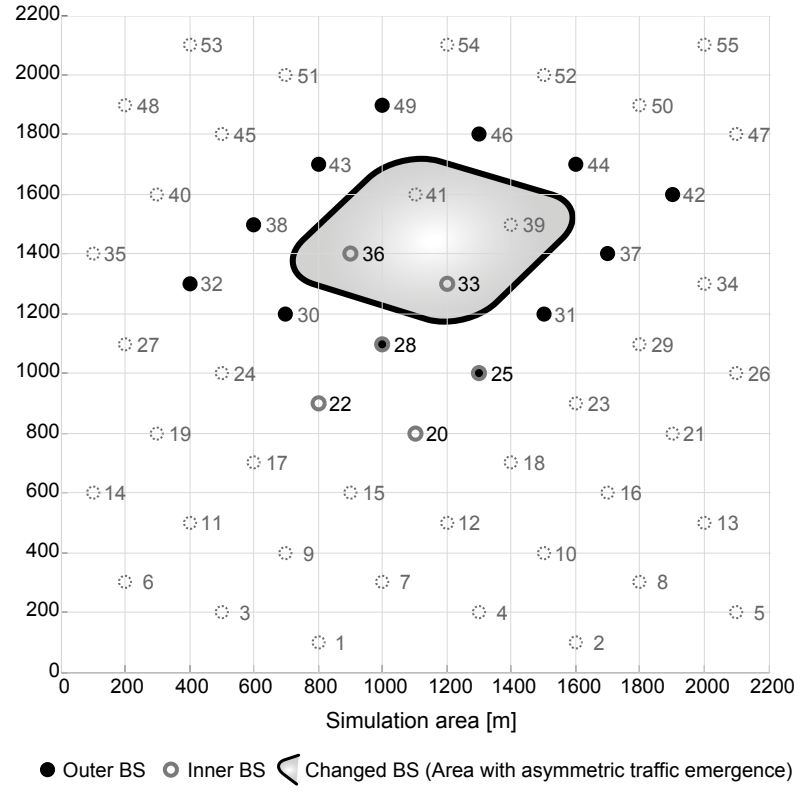

Fig. 9. Cell layout for simulations in urban environment

The cell layout was chosen according to [17, p. 53], the layout of which looks like the illustration in Figure 9. This environment is supposed to reproduce a very urban environment, with antennas below the rooftops and high buildings between them. The circles represent transmitter antennas with appropriate IDs next to them. The inner base stations which are marked with a gray circle are the ones where measurements and statistics base on. All the surrounding base stations (black circles) are there to create realistic interference. In the simulations, users move around with a turn probability of $50 \%$, which means that the probability at crossings is 0.5 for straight walk and 0.25 for each left and right turns.

Changing of link configurations and distribution of up- and downlink users is always done for the area marked in Figure $9(33,36,39$ and 41). The inner base stations are those which are analyzed, namely IDs 20, 22, 25, 28, 33 and 36 [17]. The different link configurations also affect the surrounding base stations. Therefore, also the regarding cells are evaluated in the analysis.

\section{A. Simulation Setups}

Hereafter we define two scenarios, each of which consisting of the following four different simulation setups (A - D) which are compared to each other.

1) Homogeneous and Symmetric Traffic Emergence ( setup A): By way of comparison, the first simulation is done with symmetric traffic emergence for the whole simulation area (i.e. the same amount of uplink and downlink users for each cell). Of course, throughput and BLER can still vary from cell to cell due to dissimilar distribution of users in the cell. As a TDD duplex configuration, the set-up with ID 2 (according to Figure 2) was chosen because the capacity is balanced between uplink and downlink with a relation of $4: 4$ respectively (D-UUDD-UUD, hyphen representing a special frame). Furthermore, the average latency is relatively low due to the switch periodicity of $5 \mathrm{~ms}$ in this configuration.
TABLE III

SIMUlation SERIES FOR SCENARIO 1

\begin{tabular}{|c|c|c|c|}
\hline & \multicolumn{2}{|c|}{$\varnothing$ UEs per BS } & \\
Setup & Uplink & Downlink & Duplex Configuration \\
\hline A & 10 & 10 & 2 (D-UUDD-UUD) \\
B & 5 & 15 & 2 (D-UUDD-UUD) \\
C & 5 & 15 & 3 (D-UDDD-UDD) \\
D & 5 & 15 & 5 (D-UUDDDDDD) \\
\hline
\end{tabular}

2) Inhomogeneous and Asymmetric Traffic Emergence (setup B): For the area marked in Figure 9 (changed BS), traffic emergence was now set up differently for uplink and downlink, while leaving the duplex configuration as defined in ID 2 (Figure 2). This represents an unfavorable situation for operators, where capacity is balanced equally while the demand of UEs would require a DL- or UL-heavy distribution (scenarios 1 and 2 in subsections VI-C and VI-D respectively).

3) Inhomogeneous and Asymmetric Traffic Emergence with Appropriate Duplex Configuration (setups $C$ and D): In further consequence, the link configuration is now changed accordingly so that the relation of uplink and downlink slots per frame matches the traffic emergence as good as possible. Two different configurations were simulated in each scenario.

\section{B. Example for Simulation Setup}

Table III clarifies the arrangement of UEs in scenario 1 as an example briefly. It shows the average UL and DL user amount for each setup (A - D) together with the selected link duplex configuration, as applied in scenario 1 .

\section{Scenario 1: DL-Heavy Traffic with Appropriate Duplex Configuration}

Two different link duplex configurations were set up for the area with changed BS, which are configurations 3 (D-UDDDUDD) and 5 (D-UUDDDDDD) with uplink to downlink proportion of $1: 3$ and 2:7 respectively. Figure 10 points out the simulation results for the four different combinations of link configuration and traffic emergence. Each bar represents a certain simulation setup from $\mathrm{A}$ to $\mathrm{D}$, while a group of bars shows the analysis for distinct groups of base stations, as explained former in Figure 9. Figure 10(a) shows the cell throughput for the investigated setups, while the lower part (Figure 10(b)) illustrates the user throughputs. This figure can be analyzed from different points of view.

1) Comparison of Symmetric and Asymmetric Traffic with Identical Duplex Configuration (setups $A$ and $B$ ): Initially, the differences in throughput for cells and users with identical link configuration are described. There is no difference in cell throughput, since the amount of slots is the same independent of the user amount per cell. In case of less users in a cell, each user gets more capacity, which again has no considerable impact on cell throughput. This expresses in only slight changes between line patterned and light gray bars in Figure 10(a) for both DL and UL. In contrast, user throughput is different in setup B, because of the relationship between UL and DL UEs of 1:3. This leads to more downlink users that have to be served compared to a 1:1 UE relationship, and thus to lower DL user throughput. The right part of Figure 10(b) shows the inverse effect. Less uplink users can use the 


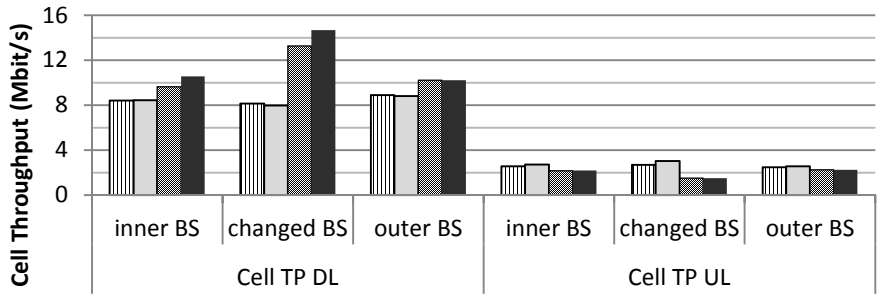

四Symm. Traffic, Conf. 2 (Setup A) $\square$ Asym. Tr., Conf. 2 (Setup B)

$\square$ Asym. Tr., Conf. 3 (Setup C) घAsym. Tr., Conf. 5 (Setup D)

(a) Cell throughput

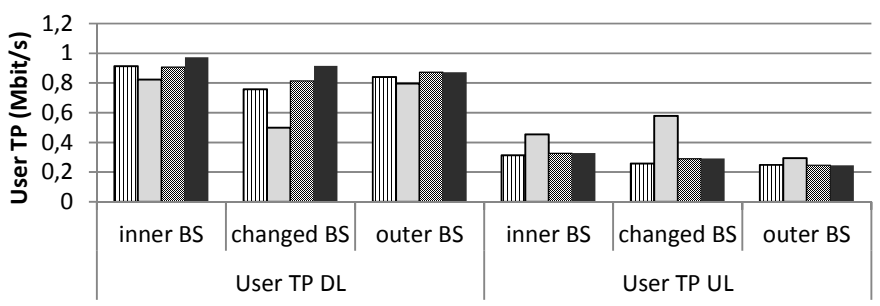

(b) User throughput

Fig. 10. Cell and User Throughput Comparison for UL and DL for scenario 1

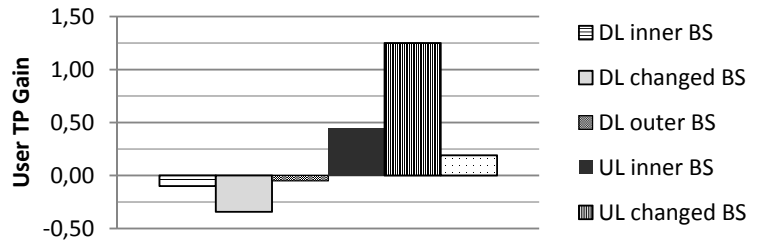

Fig. 11. Gains and losses in user throughput for different areas

same capacity compared to symmetric traffic emergence (setup B), which means higher UL user throughput. A graphical representation of the gains and losses in user throughput due to asymmetric traffic emergence is depicted in Figure 11.

2) Comparison of Asymmetric Traffic with Appropriate Duplex Configurations (setups $C$ and $D$ ): As a consequence of the asymmetric traffic emergence, the network decides to switch the TDD duplex configuration to suit the current amount of uplink and downlink users. For a traffic situation equal to setup B (section VI-C1), the duplex configuration was switched to 3 (setup C) and 5 (setup D). Both configurations have two slots reserved for uplink, while having 6 (configuration 3, setup C) and 7 (configuration 5, setup D) downlink slots. The cell throughputs were compared to the simulation with symmetrical UL-DL user distribution (setup A). Figure 12 illustrates the expected gains. The expected factors are calculated by dividing the slots reserved for the respective direction from the new configuration ( 3 or 5 ) by same of the old configuration (2). This graphic proofs that the selection of the appropriate duplex configuration does increase both performance for up- and downlink users. Especially when using configuration 3 , which matches the traffic emergence perfectly (75\% DL), considerable gains are visible. Thus, a dynamic switching point can increase the overall system performance without any need to increase the used bandwidth, power or any other parameter of the network.

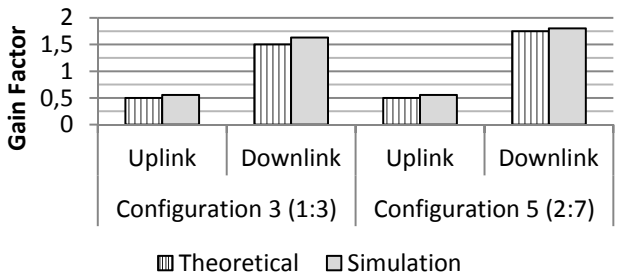

Fig. 12. Cell throughput gain of asymmetric duplex configurations for $75 \%$ of the users in DL direction

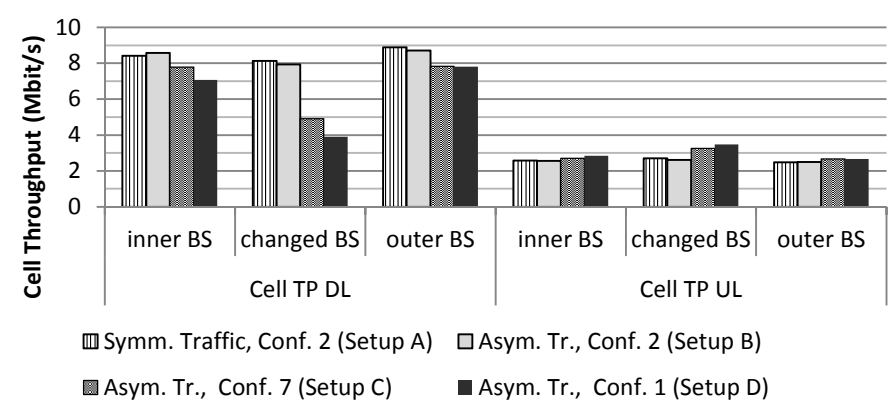

(a) Cell throughput

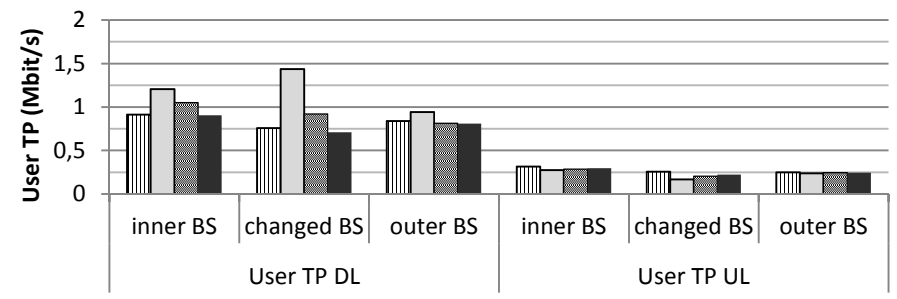

(b) User throughput

Fig. 13. Cell and User Throughput Comparison for UL and DL for scenario 2

\section{Scenario 2: UL-Heavy Traffic with Appropriate Duplex Configuration}

Analogous to former investigations in downlink-heavy scenarios, UE distribution was also changed to more uplink users in the same relation (UL:DL $=3: 1$ ). In order to adapt the link configuration to arising traffic demand, the link configurations 1 (D-UUUD-UUU) and 7 (D-UUUD-UUD) with proportions of UL:DL of 6:2 and 5:3 were used.

Figure 13 shows the simulation results for the same setups A to D, but with uplink-heavy instead of downlink-heavy traffic distribution. Changes in traffic emergence in this investigation are also done only for changed BS 33, 36, 39 and 41 (see Figure 9).

1) Comparison of Symmetric and Asymmetric Traffic with Identical Duplex Configuration (setups $A$ and B): Similar to downlink-heavy traffic scenarios, the first confrontation of symmetric and asymmetric, uplink-heavy traffic shows no considerable difference in cell throughput for both uplink and downlink direction (line patterned and light gray bars in Figure 13(a)). When looking at user throughput (Figure 13(b)), the inverse effect compared to scenario 1 occurs. The cells which serve more uplink users (changed BS) achieve a considerably lower user throughput in uplink direction due to more users which have to be handled. In contrast, due to less downlink 


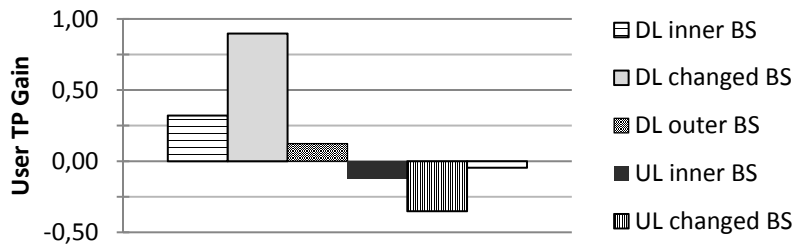

Fig. 14. Gains and losses in user throughput for different areas

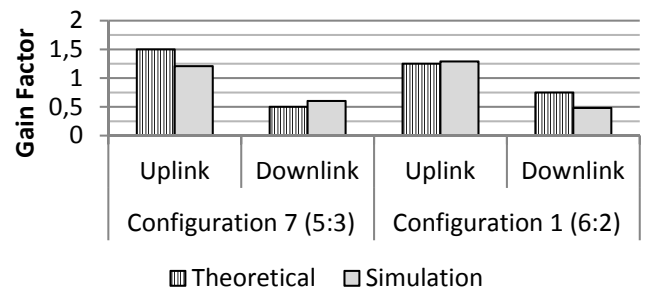

Fig. 15. Cell throughput gain of asymmetric duplex configurations for $75 \%$ of the users in UL direction

users therein, the user throughput of those can be increased (setup B).

Figure 14 illustrates the gains and losses in user throughput in case of an uplink-heavy traffic without changing the link configuration. Again, the opposite effect compared to Figure 11 in scenario 1 shows up, with a huge gain in downlink direction (light gray bar) and a loss in uplink (horizontal line patterned bar).

2) Comparison of Asymmetric Traffic with Appropriate Duplex Configurations (setups $C$ and $D$ ): To analyze the results of matching the duplex configuration to traffic for the affected cells, the dark gray and black bars of Figure 13 are focused. Configurations 7 (setup C) and 1 (setup D) are selected to match the uplink-heavy traffic situation in scenario 2. As a consequence, the cell throughput in downlink direction decreases significantly for the cells with asymmetric traffic (changed BS in Figure 13(a)). In contrast, cell throughput rises for uplink users by up to $35 \%$ when switching from configuration 2 to 1 . Due to the very same simulation setup of both simulations, this effect can be fully affiliated to the change of the TDD duplex configuration. Figure 13(b) shows that user throughput of uplink users rises when selecting a matching duplex configuration (setups $\mathrm{C}$ and D) compared to asymmetric traffic with a symmetric duplex configuration (setup B). In downlink direction, gains appear for setup C due to 3 downlink slots, while the situation is nearly equal to setup A if configuration 1 is in use ( 2 downlink slots). As done for scenario 1, relative gains and losses in cell throughput are compared as theoretically calculated values and simulation results, and are illustrated in figure 15 . The gain is not as decisive as in scenario 1 (Figure 12), but shows also losses in case of less slots for the respective direction.

\section{CONCLUSION}

We propose an LTE simulator which is capable of analyzing how system performance changes as soon as the emerging traffic deviates from the available capacity. A detailed description of the used system model is given. As a consequence of the changing demands on capacity distribution between uplink and downlink, this investigation shows that an adaptive switching point through changing of the TDD link duplex configuration can react better to downlink-heavy than uplinkheavy situations. However, an average user throughput which is rather insensitive against the influence of other users' behavior can be achieved. The reason behind that is a better utilization and distribution of the available bandwidth to all users compared to a fixed switching point. This is beneficial for both end-users and operators. A more balanced speed can be provided for customers, because the network can react to bottlenecks. Operators may use their infrastructure more efficiently. The benefits are quantified within this study.

\section{REFERENCES}

[1] G. Piro, L. Grieco, G. Boggia, F. Capozzi, and P. Camarda, "Simulating LTE cellular systems: An open-source framework," IEEE Transactions on Vehicular Technology, vol. 60, no. 2, pp. 498-513, Feb. 2011.

[2] J. Ikuno, M. Wrulich, and M. Rupp, "System level simulation of LTE networks," in Vehicular Technology Conference (VTC 2010-Spring), 2010 IEEE 71st, May 2010, pp. 1-5.

[3] Q. Zheng, H. Du, J. Li, W. Zhang, and Q. Li, "Open-LTE: An open LTE simulator for mobile video streaming," in 2014 IEEE International Conference on Multimedia and Expo Workshops, Jul. 2014, pp. 1-2.

[4] Y. Wang, S. Frattasi, T. Sorensen, and P. Mogensen, "Network timesynchronization in TDD based LTE-advanced systems," in Vehicular Technology Conference, 2009. VTC Spring 2009. IEEE 69th, Apr. 2009, pp. 1-5.

[5] Y. Wang, K. Valkealahti, K. Shu, R. Sankar, and S. Morgera, "Performance evaluation of flexible TDD switching in 3gpp LTE system," in 2012 35th IEEE Sarnoff Symposium (SARNOFF), May 2012, pp. 1-4.

[6] J. R. Kulcsar, "Qualitätsevaluierung repräsentativer Werte für das Interface zwischen Link-Level- und System-Level-Simulatoren in Mobilkommunikationssystemen," Master's thesis, Fachhochschule Hagenberg, Austria, 2009.

[7] J. C. Ikuno, M. Wrulich, and M. Rupp, "System level simulation of LTE networks," in Proc. 2010 IEEE 71st Vehicular Technology Conference: VTC2010-Spring, Taipei, May 2010.

[8] J. B. Andersen, T. S. Rappaport, and S. Yoshida, "Propagation measurements and models for wireless communications channels," Comm. Mag., vol. 33, no. 1, pp. 42-49, Jan. 1995.

[9] J.-E. Berg, "A recursive method for street microcell path loss calculations," in Personal, Indoor and Mobile Radio Communications, 1995. PIMRC'95. 'Wireless: Merging onto the Information Superhighway'. Sixth IEEE International Symposium on, vol. 1, 1995, pp. 140-143.

[10] C. Osterkorn, G. Ostermayer, and M. Huemer, "Comparison of oneand two-dimensional slow fading models in mobile radio system simulations," in IEEE 16th International Symposium on Personal, Indoor and Mobile Radio Communications, 2005. PIMRC 2005, vol. 4, Sep 2005, pp. 2650-2654 Vol. 4.

[11] T. T. Ha, Theory and Design of Digital Communication Systems. Cambridge University Press, 2010.

[12] E. Dahlman, S. Parkvall, J. Skold, and P. Beming, $3 G$ Evolution, Second Edition: HSPA and LTE for Mobile Broadband. Academic Press, 2008.

[13] V. Pauli, I. Viering, C. Buchner, E. Saad, G. Liebl, and A. Klein, "Efficient link-to-systemlevel modeling for accurate simulations of MIMO-OFDM systems," in Proceedings of the 2009 IEEE international conference on Communications, ser. ICC'09. Piscataway, NJ, USA: IEEE Press, 2009, pp. 3599-3604.

[14] M. Wrulich, S. Eder, I. Viering, and M. Rupp, "Efficient Link-to-System Level Model for MIMO HSDPA," in IEE GlobeCom. IEEE Press, 2008

[15] A. G. Alexiou, C. Bouras, V. Kokkinos, A. Papazois, and G. Tsichritzis, "Efficient MCS selection for MBSFN transmissions over LTE networks." in Wireless Days. IEEE, 2010, pp. 1-5.

[16] 3GPP, "Evolved Universal Terrestrial Radio Access (E-UTRA); Physical layer procedures," 3rd Generation Partnership Project (3GPP), TS 36.213, Sep. 2008

[17] ETSI, "Universal Mobile Telecommunications System (UMTS); Selection procedures for the choice of radio transmission technologies of the UMTS," ETSI, Tech. Rep., 1998, tR 101112 V3.2.0 (1998-04). 